\title{
Asymptotic shape of the region visited by an Eulerian Walker
}

\author{
Rajeev Kapri斑 and Deepak Dhar \\ Department of Theoretical Physics, Tata Institute of Fundamental Research, \\ 1 Homi Bhabha Road, Colaba, Mumbai - 400 005, India.
}

(Dated: June 10, 2018)

\begin{abstract}
We study an Eulerian walker on a square lattice, starting from an initial randomly oriented background using Monte Carlo simulations. We present evidence that, for large number of steps $N$, the asymptotic shape of the set of sites visited by the walker is a perfect circle. The radius of the circle increases as $N^{1 / 3}$, for large $N$, and the width of the boundary region grows as $N^{\alpha / 3}$, with $\alpha=0.40 \pm .06$. If we introduce stochasticity in the evolution rules, the mean square displacement of the walker, $\left\langle R_{N}^{2}\right\rangle \sim N^{2 \nu}$, shows a crossover from the Eulerian $(\nu=1 / 3)$ to a simple random walk $(\nu=1 / 2)$ behaviour.
\end{abstract}

PACS numbers: 05.50.+q, 02.50.-r

\section{INTRODUCTION}

There has been a lot of interest in the study of asymptotic shape of growing clusters. One of the earliest of such studies was by Richardson, who showed that the asymptotic shape of the infected region in an epidemic model has linear segments [1]. In the Eden model [2], which models an epidemic without recovery, it has been shown that the asymptotic shape of the growing cluster is not a perfect circle [3]. The shape of growing clusters has also been studied in sandpile models. In the abelian sandpile model, in two dimensions in an initial background of $h$ particles at each lattice site [4, 5], it was found that the cluster in general has a convex asymptotic shape, which becomes more circular as $h$ is decreased, and tends to a perfect circle as $h \rightarrow-\infty$.

In this paper, we study the Eulerian walker (EW) model on the square lattice. This model is related to the sandpile model and was initially introduced by Priezzhev et al. [6, 7] as a simpler variant of the sandpile model of self-organized criticality (SOC) 8]. It has subsequently found applications in design of derandomized simulations of Markov chains 9], efficient information transfer protocols in computer networks [10], and modelling coevolution of virus and immune systems [11]. We study the model, starting from a disordered background, by Monte Carlo simulations. We have a single walker that moves on the lattice and we look at the shape of region visited by it which grows with the length $N$ of the walk. Interestingly, we find evidence that this region is asymptotically a perfect circle. The circular shape is reminiscent of the circular shape in the rotor-router aggregation model studied by Propp (see [12, 13]), where, for a special initial configuration, the region is almost a perfect circle [14] with

\footnotetext{
*Present address: Department of Physics, Indian Institute of Science Education and Research Mohali, MGSIPAP Complex, Sector 26, Chandigarh - 160 019, India.

${ }^{\dagger}$ Electronic address: rkapri@theory.tifr.res.in

‡Electronic address: ddhar@theory.tifr.res.in
}

departures from the circle being of order 1 . The circular shape for the EW cluster is not very evident for small walks. For example, in Fig. 1] we have shown clusters formed by the EW of $N=10^{5}$ and $10^{7}$ steps. Clearly, only for large $N$, does the circular shape start to emerge, and it requires careful statistical analysis to see this when $N$ is not so large.

The EW model can also be looked upon as a particular
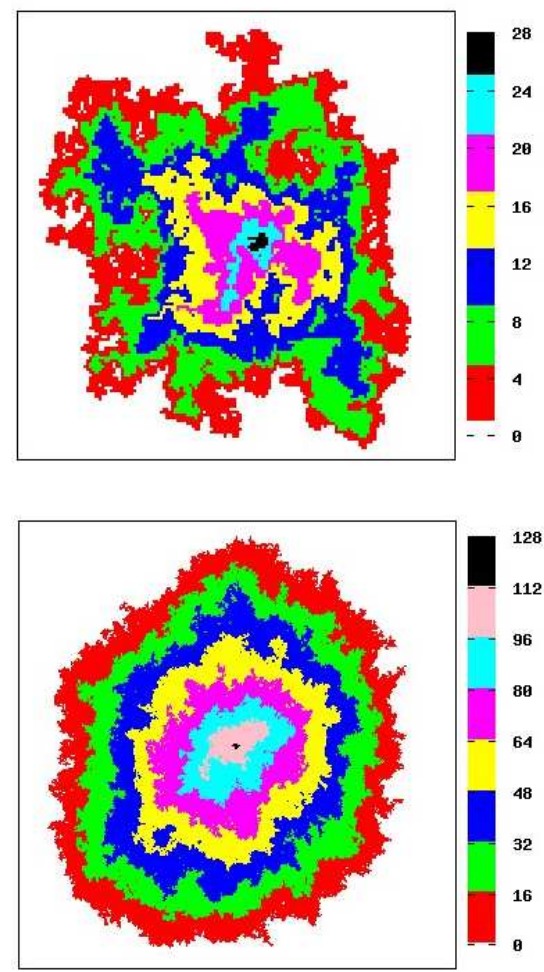

FIG. 1: (Color online) Cluster formed by an EW after $10^{5}$ (top) and $10^{7}$ (bottom) steps. A site is assigned a color depending on whether the numbers of visits to it is in the range $1-4,5-8$ etc. for $10^{5}$, and $1-16,17-32$ etc. for $10^{7}$ steps (key panel displayed on the right). The diameters of the clusters are 134 and 600 units respectively. 


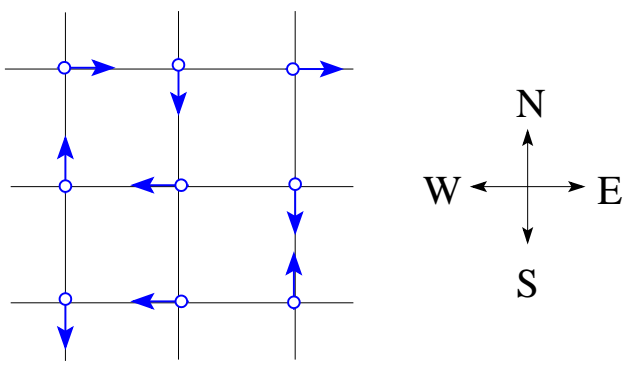

FIG. 2: (Color online) A portion of the square lattice. At each lattice site, the outgoing bond is shown by the arrow.

limit of a growing self repelling walk, in which the walker preferably jumps along a bond which has been visited least number of times so far. This model was studied in $1 d$ by Toth and Veto [15]. In the zero temperature limit, in one specific variant, this becomes the EW model and a finite temperature corresponds to noise. It was found that the number of visits at a distance $y$ from the origin satisfies the scaling function $F(y)=1-y$, for $0 \leq y \leq$ 1 . We find that the same scaling function holds even in $2 d$. We also study the model in the presence of noise, where there is a small probability $\epsilon$ that walker goes in a direction not given by the EW rule. We find that a small noise changes the asymptotic behaviour. The diameter of growing region scales as $A(\epsilon) N^{1 / 2}$ in the presence of noise and as $N^{1 / 3}$ in its absence.

The paper is organized as follows. In Sec.[II we define our model. In Sec. III, we give details of the simulation without noise (i.e. $\epsilon=0$ ). We find that the asymptotic shape of lines of constant average number of visits by an EW are perfectly circular, within statistical errors. We will argue that the variance of average number of visit at any distance from the origin tends to a finite number for large $N$ and also obtain the scaling function for the average number of visits. In Sec. IV we discuss the case with noise, and finally we summarize our results in Sec. V]

\section{MODEL}

The Eulerian walker is defined as follows: We consider a square lattice. We associate with each site an arrow which can point to along one of the four directions, denoted by N, E, S and W (Fig. 22). In the initial configuration, the direction of the arrow at each site is chosen independently, and with equal probability. We put a walker at the origin which moves on the lattice. The motion of the walker is affected by configuration of arrows on the lattice, which in turn affects the arrow configuration on the lattice. The walker follows the following rule: at each time step, the walker after arriving at a site rotates the arrow at that site in a clockwise direction by $90^{\circ}$, and then moves one step along the new arrow direction.

It was shown in Ref. [6] that on any finite graph, us-

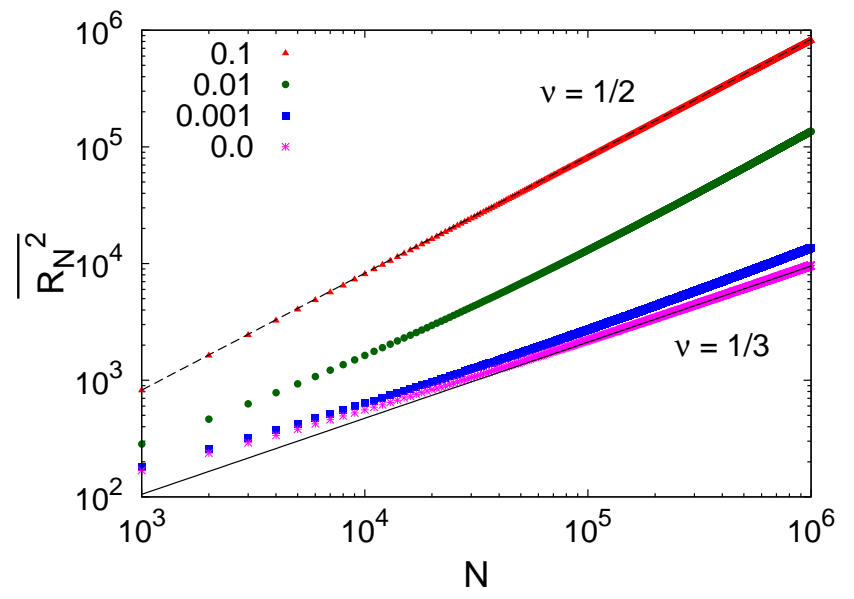

FIG. 3: (Color online) Mean square displacement $\overline{R_{N}^{2}}$ of the walker from the origin as a function of $N$ for various $\epsilon$ values. For the pure case (i.e. $\epsilon=0) \overline{R_{N}^{2}}$ grows as $N^{2 \nu}$ with $\nu=1 / 3$. In the presence of noise, there is a cross over from the Eulerian $(\nu=1 / 3)$ to a simple random walk behaviour $(\nu=1 / 2)$.

ing the above rules, the walker eventually visits all sites and settles into a limit cycle which is an Eulerian circuit visiting each directed bond exactly once in a cycle. This is not the case on an infinite lattice, where the walker always finds new bonds which are not visited earlier and the number of visited sites keeps on growing. It was noted already that in $2 d$, the diameter of the region visited by the walker grows as $N^{1 / 3}$, but the asymptotic shape was not investigated.

The EW with noise is defined as follows: at each time step the walker rotates the arrow at its location by $0^{\circ}$, $90^{\circ}, 180^{\circ}$ or $270^{\circ}$ with probability $\epsilon / 3,1-\epsilon, \epsilon / 3$ or $\epsilon / 3$ respectively. We will show that the diameter of the region visited grows as $N^{1 / 2}$ for nonzero $\epsilon$ and as $N^{1 / 3}$ when $\epsilon=0$.

\section{NUMERICAL SIMULATIONS FOR EVOLUTION WITHOUT NOISE}

First we discuss the case $\epsilon=0$. We denote the number of times different sites visited by the walker after $N$ steps by $n_{N}(\mathbf{x})$ and the walker's square displacement from the origin by $R_{N}^{2}$. We evaluate $\overline{n_{N}}(\mathbf{x})$ (the over line represents averaging over initial conditions), the variance of $n(\mathbf{x})$ denoted by $\operatorname{Var}\left[n_{N}(\mathbf{x})\right]$, and the mean square displacement $\overline{R_{N}^{2}}$ by averaging over $10^{6}$ different initial configurations.

\section{A. Mean square displacement}

According to the heuristic argument given in Ref. [6], if at time $t$ the number of sites visited by the walker is $S(t)$, then in the previous $4 S(t)$ time steps, most of these 
sites have been visited exactly 4 times except a small fraction at the boundary. As the cluster is seen to have few holes, it is nearly compact, and $S(t) \sim D^{2}(t)$, where $D(t)$ is the diameter of the cluster, at time $t$. Thus we get

$$
\frac{d D(t)}{d t} \sim \frac{1}{D^{2}}
$$

which implies that after $N$ steps,

$$
D_{N} \sim N^{\nu} \quad \text { with } \nu=\frac{1}{3}
$$

Figure 3 shows the mean square displacement of the $\mathrm{EW}$ as a function of its length $N$. The averaging is done over $10^{6}$ realizations. The straight line, which is the best fit to the data has a slope $0.33 \pm 0.01$, consistent with Eq. (2).

\section{B. Average number of visits}

As seen in Fig. 1, the cluster of visited sites is quite irregular in shape. Also sites that have been visited at least $n$ times have rough boundaries with several islands of sites that have been visited fewer number of times than all the surrounding sites. However, if we average over different realizations of the initial arrow configuration, some interesting regularities are seen.

In Fig. 4, we have plotted lines of $\overline{n_{N}}(\mathbf{x})=\zeta$, for different $\zeta$ as indicated in the figure, for an EW of length $N=10^{6}$ averaged over $10^{7}$ realizations. To obtain these lines, we add a diagonal bond between $(x, y)$ and $(x+1, y+1)$ for each $(x, y)$, and extend the definition of $\overline{n_{N}}(\mathbf{x})$ to all real $\mathbf{x}$ by linear interpolation within each small triangle. The plot shows that these lines are nearly perfect circular in shape.

The shape of rings, for large $N$, can be defined by a function

$$
f(\theta)=\lim _{N \rightarrow \infty} \frac{r_{N}(\theta)}{N^{1 / 3}},
$$

where $r_{N}(\theta)(0 \leq \theta<2 \pi)$ is the angle dependent radius. If the shape is a perfect circle $f(\theta)=$ constant, otherwise $f(\theta)$ is a periodic function of $\theta$ that can be expressed in terms of Fourier cosine series

$$
f(\theta)=\sum_{m=0}^{\infty} a_{4 m} \cos (4 m \theta) .
$$

Since the shape has fourfold symmetry the series will only have terms with $m=4 u(u=0,1,2 \ldots)$. The vanishing of $a_{4 m}$ 's for all $m \neq 0$ then implies a circular shape. We define

$$
A_{4}(r)=\frac{\sum_{j} \overline{n_{N}}\left(\mathbf{x}_{j}\right) \cos \left(4 \theta_{j}\right)}{\sum_{j} \overline{n_{N}}\left(\mathbf{x}_{j}\right)}
$$

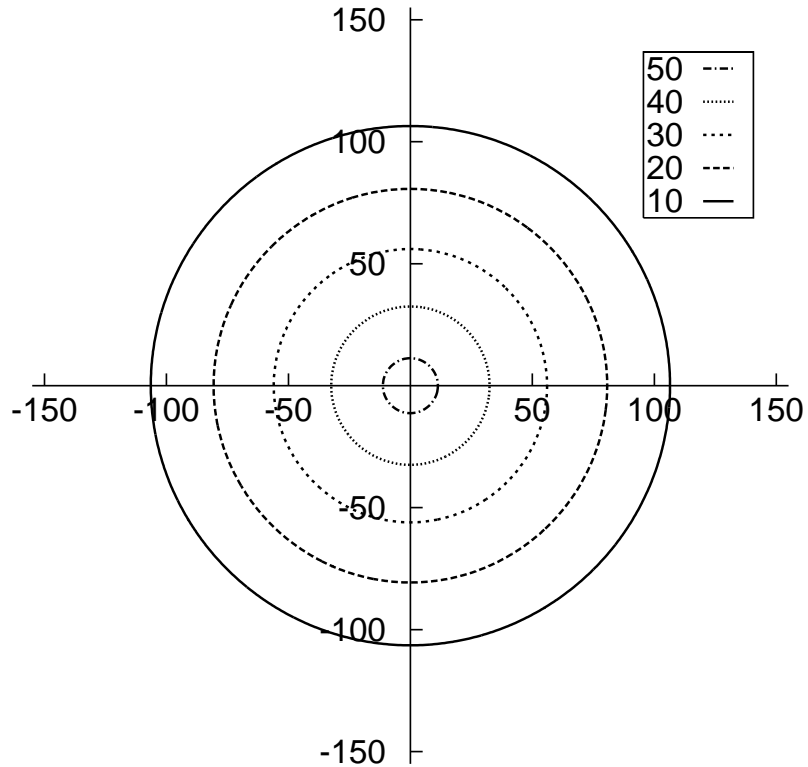

FIG. 4: Contours of the constant average number of visits, $\overline{n_{N}}(\mathbf{x})$ for various values as indicated, for the $\mathrm{EW}$ of $N=10^{6}$ steps averaged over $10^{7}$ samples.

as the normalized amplitude of the fourth Fourier mode. The summation $j$ is over all the lattice points whose Euclidean distance from the origin lies between $r$ and $r+1$. Here $\theta_{j}$ is the angle that the vector $\mathbf{x}_{j}$ makes with the $x$-axis. This function, for a fixed $r$, has a well defined limit for $N \rightarrow \infty$. In Fig. 國(a), we have shown $A_{4}(r)$ as a function of $r$ for $N=10^{6}$ steps. The plot shows that for large $r, A_{4}(r)$ approaches zero with fairly large irregularlooking fluctuations. These fluctuations, for a fixed $r$, do not become smaller by statistical averaging or larger $N$. These fluctuations occur because the lattice points lying between radii $r$ and $r+1$ are not distributed perfectly evenly along the ring. They are of number-theoretic origin, and have been studied in the mathematics literature under the name of the 'Gauss circle problem' 17]. The analysis of moments of $A_{4}$ is therefore not very useful to estimate the shape and we have to adopt some other procedure.

The simplest way to have a quantitative estimate of shape of rings is to fit the data and obtain the mean radius for various rings of constant $\overline{n_{N}}(\mathbf{x})$. In Fig. [5), we have shown such a ring and its fitting for $\overline{n_{N}}(\mathbf{x})=1$. The best fit gives the mean radius $\langle R\rangle=145.436 \pm 0.003$ for $N=10^{6}$. For other rings also we find the error bars of the same order showing that the line of constant average number of visits are circular in shape within an error bar of $0.002 \%$. The inset shows a close up of a particular region of the ring.

We also calculate the root mean square deviation of distance, $\Delta r(\zeta)$, of points on the line of constant $\overline{n_{N}}(x)=$ $\zeta$ to the origin with mean radius $\langle R\rangle_{\zeta}$. This is shown in 


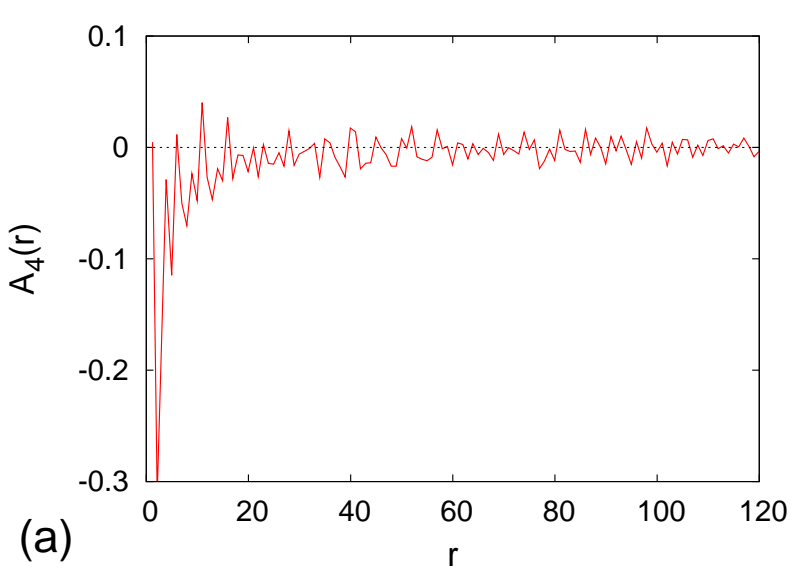

(b)
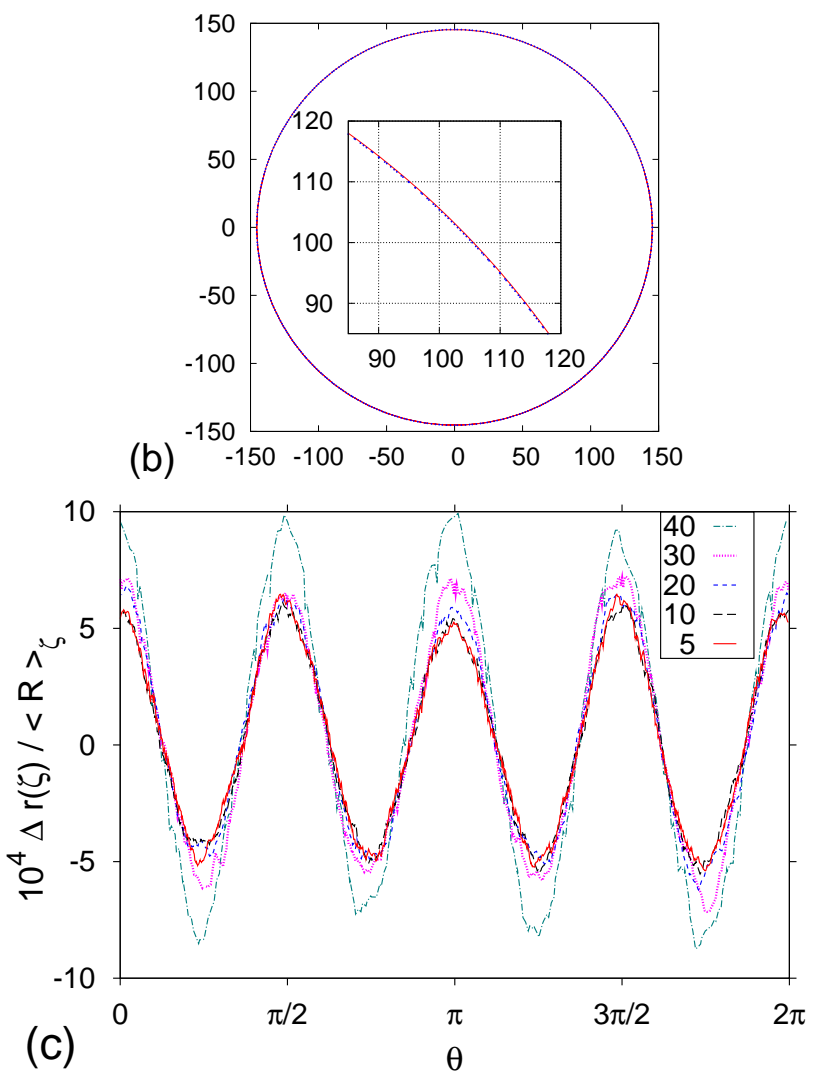

FIG. 5: (Color online) (a) $A_{4}(r)$ vs $r$ for $N=10^{6}$ steps. (b) Contour of $\overline{n_{N}}(\mathbf{x})=1$ and its fit. The inset zooms a particular portion of the curve. (c) $\Delta r(\zeta) /\langle R\rangle_{\zeta}$ as a function of $\theta$ for various $\overline{n_{N}}(\mathbf{x})=\zeta$ values.

Fig. 罒(c) as a function of $\theta$ for various $\zeta$. The plot shows that, $\Delta r(\zeta) /\langle R\rangle_{\zeta}$ is of the order $10^{-4}$ and decreases as $\langle R\rangle_{\zeta}$ increases.

Another way to estimate the shape of the cluster formed by visited sites is to obtain various moments of the data. Since all the four directions are equivalent for the walker, we expect that $\overline{n_{N}}(\mathbf{x})$ has a fourfold symmetry. For a given length $N$, we calculate $\left\langle x^{4}\right\rangle,\left\langle y^{4}\right\rangle$ and $\left\langle x^{2} y^{2}\right\rangle$ moments. If the shape of the cluster is perfect circular we would have

$$
\frac{\left\langle x^{4}\right\rangle}{\left\langle x^{2} y^{2}\right\rangle}=\frac{\left\langle y^{4}\right\rangle}{\left\langle x^{2} y^{2}\right\rangle}=3 .
$$

For $N=10^{6}$ steps averaged over $10^{6}$ initial realizations, we find that $\left\langle x^{4}\right\rangle /\left\langle x^{2} y^{2}\right\rangle=\left\langle y^{4}\right\rangle /\left\langle x^{2} y^{2}\right\rangle=3.007$, which is consistent with the asymptotic value 3 deviation being only about $0.2 \%$.

\section{Scaling of $\overline{n_{N}}(\mathrm{x})$}

For large $N, \overline{n_{N}}(\mathbf{x})$, the average number of visits to the site $\mathbf{x}$, depends only on $|\mathbf{x}|$. Therefore, we expect that $\overline{n_{N}}(\mathbf{x})$, satisfies the scaling form

$$
\overline{n_{N}}(|\mathbf{x}|)=a N^{1 / 3} F\left(\frac{|\mathbf{x}|}{b N^{1 / 3}}\right),
$$

where $F(y)$ is the scaling function.

The scaling function can be determined as follows: Let $\mathbf{x}_{1}$ and $\mathbf{x}_{2}\left(\left|\mathbf{x}_{2}\right|>\left|\mathbf{x}_{1}\right|\right)$ be the distances of two different sites from the origin. The walker would have made several visits to $\mathbf{x}_{1}$ before it first reaches $\mathbf{x}_{2}$. Afterwards, because of the local Euler-like organization of the arrows, both sites are visited equally often. Therefore, the difference between the number of times sites at distances $\mathbf{x}_{\mathbf{1}}$ and $\mathbf{x}_{2}$ are visited remains bounded as $N \rightarrow \infty$, i.e.,

$$
\overline{n_{N}}\left(\mathbf{x}_{1}\right)-\overline{n_{N}}\left(\mathbf{x}_{2}\right)=a N^{1 / 3}\left[F\left(y_{1}\right)-F\left(y_{2}\right)\right]=\text { constant } .
$$

This implies that $F(y)$ must be a linear function of $y$. Using the freedom of choice of constants $a$, and $b$, we can set $F(0)=1$ and $F(1)=0$. Therefore, we have

$$
F(y)=\left\{\begin{array}{l}
1-y \text { for } 0 \leq y \leq 1 \\
0 \text { otherwise }
\end{array}\right.
$$

This simple form of $F(y)$ was already noted by Toth and Veto for the problem in one dimension [15, 16]. The normalization condition $\int \overline{n_{N}}(\mathbf{x}) d \mathbf{x}=N$ gives $a b^{2}=3 / \pi$.

In Fig. 6, we have plotted the finite size scaling of $\overline{n_{N}}\left(x \mathbf{e}_{x}\right)$ for $N=10^{6}, 10^{7}$ and $10^{8}$ steps with $a=0.5$ and $b=1.38$. In the same plot, we have also shown the scaling form given by Eq. (91). The plot shows that as $N$ is increased, the scaled data approaches rather slowly towards the scaling form. In particular for smaller $|\mathbf{x}|$, the approach to the asymptotic curve seems to be slow.

\section{Variance of $n_{N}(\mathbf{x})$}

We also monitored the variance of $n_{N}(\mathbf{x})$ as a function of $N$ for different $|\mathbf{x}|$. This is plotted in Fig. 7 The graph shows that $\operatorname{Var}\left[n_{N}(\mathbf{x})\right]$ increases slowly with $N$ and suggests that $\operatorname{Var}\left[n_{N}(\mathbf{x})\right]$ remains finite for all fixed $\mathbf{x}$. This can be understood as follows: the variance of $n_{N}(\mathbf{x})$ arises only from the randomness in the initial visits 


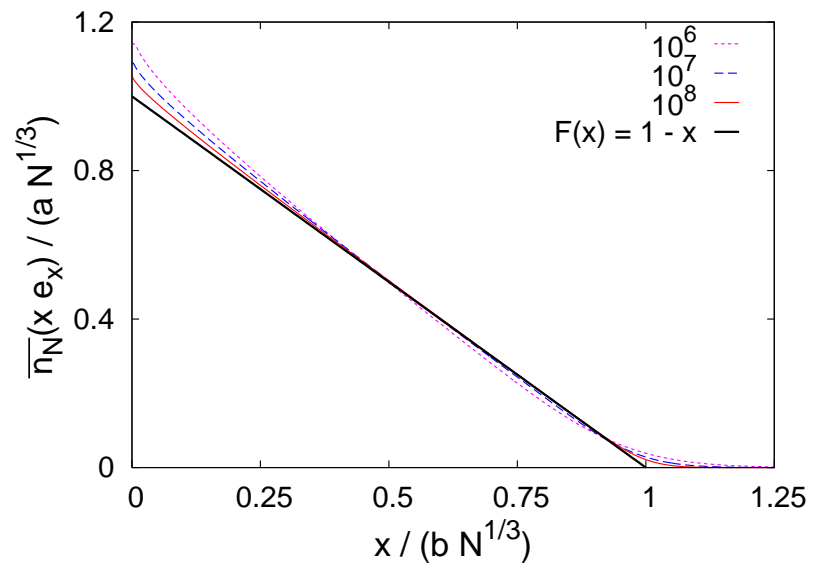

FIG. 6: (Color online)Data collapse of $\overline{n_{N}}\left(x \mathbf{e}_{x}\right)$ for $N=$ $10^{6}, 10^{7}$ and $10^{8}$ steps. The thick solid line is the scaling function.

of walker to $\mathbf{x}$. Once the local bonds have been organized into a near Euler circuit, the subsequent increments in $n_{N}(\mathbf{x})$ are nearly deterministic.

\section{E. Roughness exponent}

We define the surface of the set of visited sites as all visited sites that have at least one unvisited neighbor. Let $W_{N}^{2}$ denotes the variance of the distance from the origin of randomly picked surface formed by the EW of length $N$. Then, $W_{N}^{2}$ is defined by

$$
W_{N}^{2}=\overline{\frac{1}{M} \sum_{i=1}^{M}\left(\left|\mathbf{x}_{i}\right|-\langle R\rangle\right)^{2}},
$$

where $M$ is the number of surface points $\left|\mathbf{x}_{i}\right|$ of the cluster, $\langle R\rangle$ is the average distance of a perimeter site, and

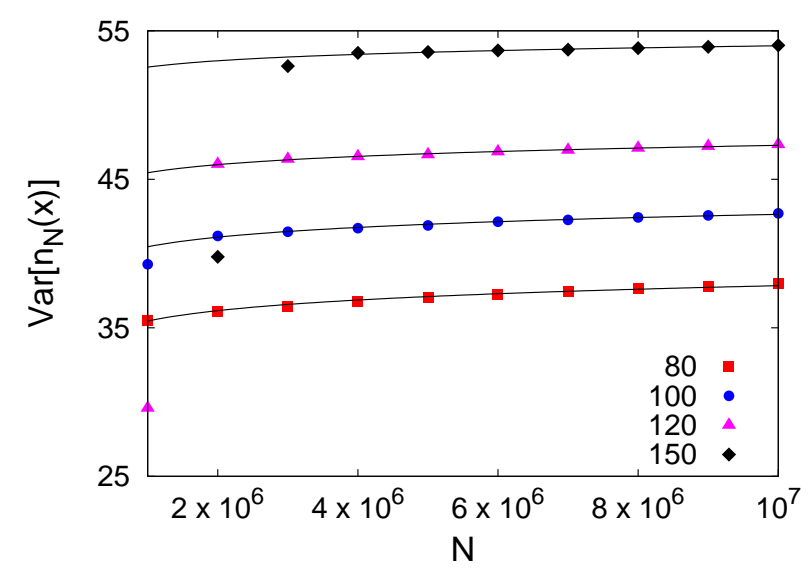

FIG. 7: (Color online) $\operatorname{Var}\left[n_{N}(\mathbf{x})\right]$ as a function of length $N$ for various $|\mathbf{x}|$. The lines are the best fit to the data.

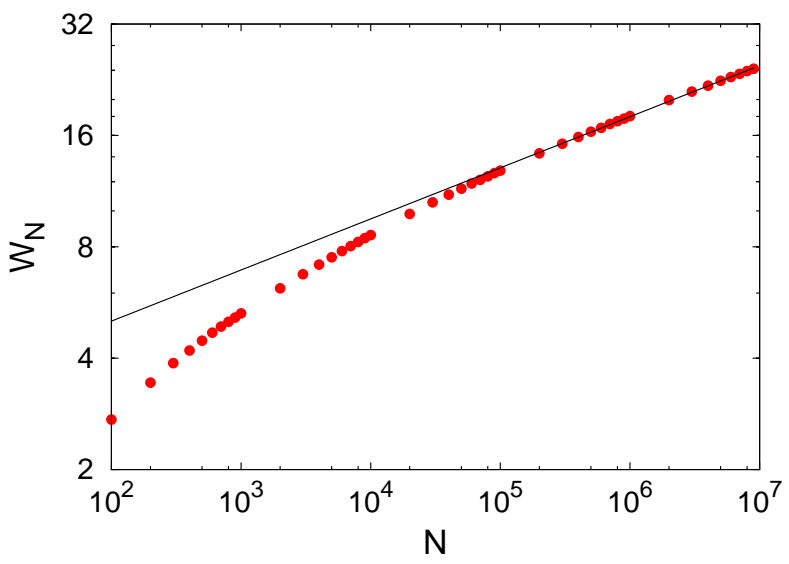

FIG. 8: (Color online) The width, $W_{N}$, of the boundary of the cluster formed by the EW vs its length $N$. The data is averaged over $10^{4}$ samples. The straight line is the best fit to the data with slope $\delta=0.136 \pm 0.002$.

the overbar denotes averaging over different clusters. We define the width of the surface by square root of $W_{N}^{2}$.

In Fig. 8 we have plotted $W_{N}$ for various $N$, in a $\log$ $\log$ scale, averaged over $10^{4}$ different clusters. We observe that $W_{N} \sim N^{\delta} \sim R^{3 \delta} \sim R^{\alpha}$ with $\delta=0.136 \pm 0.02$, which gives the roughness exponent $\alpha=0.40 \pm 0.06$. The effective value of $\alpha$ seems to decrease with $N$, and it is difficult to estimate its limiting value. It is consistent with the asymptotic value $1 / 3$ expected for the KardarParisi-Zhang (KPZ) surface growth process [18]. Note that in order to think of growth of cluster of visited sites as a local growth process, we have to redefine time so that the radius of the cluster grows linearly in the new variable.

\section{NUMERICAL SIMULATIONS FOR EVOLUTION WITH NOISE}

For the evolution with noise also, we monitored $\overline{R_{N}^{2}}$ and $\overline{n_{N}}(\mathbf{x})$ for various $N$ by averaging over $10^{6}$ different initial configurations.

\section{A. Mean square displacement}

For zero noise, for $N<10^{3}, \overline{R_{N}^{2}}$ increases roughly linearly with $N$ and then as $N^{2 / 3}$. In Fig. 3, we have also shown $\overline{R_{N}^{2}}$ as a function of $N$ for noise strengths $\epsilon=0.001,0.01$, and 0.1 . For $\epsilon=0.001$, there is only a small change from the Eulerian like behaviour. However, when the noise strength is increased, there is a clear crossover seen in $\overline{R_{N}^{2}}$ from the Eulerian like to a simple random walk behaviour, i.e. $\overline{R_{N}^{2}} \sim N$. This crossover can be observed even for noise strength as small as $(\epsilon=0.01)$. That presence of noise changes the critical behaviour is 


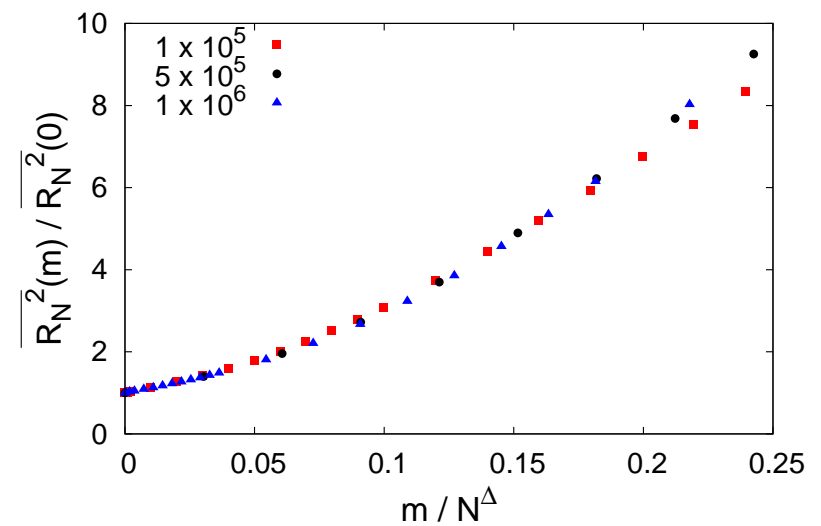

FIG. 9: (Color online) $\overline{R_{N}^{2}}(m) / \overline{R_{N}^{2}}(0)$ vs $m / N^{\Delta}$ with $\Delta=$ $0.74 \pm 0.01$ for various $N$.

not so unexpected. In equilibrium critical phenomena, the well known Harris criterion [19] characterizes a large class of systems where the critical behavior is substantially altered by the presence of disorder. In SOC models, the Manna model [20] with stochastic toppling rules is in a different universality class than the model with deterministic toppling rules (e.g., the Bak-Tang-Wiesenfeld model) 21]. In fact, different types of stochasticity can yield different universality classes. For example, in the directed sandpile with stickiness one gets a different behaviour than the stochastic Manna model (for the undirected case the situation is less clear 22, 23] ). It is known that in some sandpile models, adding noise can change the transition from first order to continuous [24].

Let $m=N \epsilon$ be the number of 'mistakes' the walker makes in a walk of $N$ steps and let $\overline{R_{N}^{2}}(m)$ represents the mean square displacement of a walk with $m$ mistakes. We would like to know how $\overline{R_{N}^{2}}(m)$ increases with $m$. Consider the effect of a single mistake. The rules of the Eulerian walk are such that as the walk evolves, the initial random arrow-directions are rearranged into an Euler circuit gradually. A wrong step would disrupt an evolving local Eulerian circuit. This local defect can be repaired, and a possibly different locally Euler-like circuit formed, when the walker revisits the site. Therefore, there is not much change in $\overline{R_{N}^{2}}(m)$ for small $m$. However, if $m$ is larger (i.e., of the order $N$ ), the walker keeps on making new mistakes before the old mistakes can be corrected, and the self-organization into Eulerian circuits is lost. This suggests that there should be a value $m^{*}$, such that walks are nearly Eulerian for $m<m^{*}$, and random walk-like for $m>m^{*}$. We find that $m^{*} \sim N^{\Delta}$ with $\Delta<1$. Furthermore, we find that $\overline{R_{N}^{2}}(m)$ satisfies a scaling relation

$$
\overline{R_{N}^{2}}(m)=\overline{R_{N}^{2}}(0) G\left(\frac{m}{N^{\Delta}}\right),
$$

where $G(x)$ is the scaling function. In Fig. 9, we have plotted $\overline{R_{N}^{2}}(m) / \overline{R_{N}^{2}}(0)$ vs $m / N^{\Delta}$ for $N=1 \times 10^{5}, 5 \times 10^{5}$

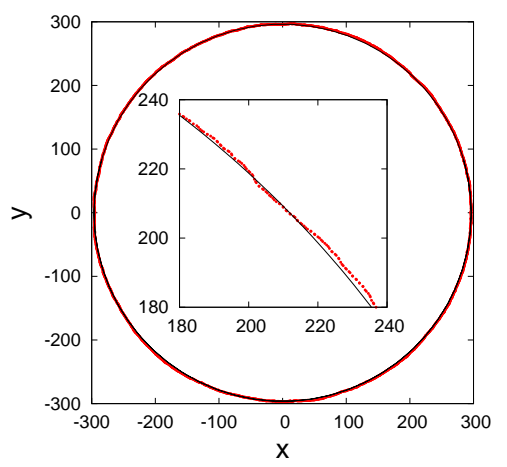

FIG. 10: (Color online) Contour of $\overline{n_{N}}(\mathbf{x})=1$ for the EW of $N=10^{5}$ steps in the presence of noise $(\epsilon=0.01)$. The averaging is done over $10^{6}$ samples. The line is the best fit to the data.

and $1 \times 10^{6}$ steps. A good collapse is obtained for $\Delta=0.74 \pm 0.01$. Equation (11) can also be written as (using $\epsilon N=m) \overline{R_{N}^{2}}(\epsilon)=\overline{R_{N}^{2}}(0) G\left(\epsilon N^{1-\Delta}\right)$. Then we have $\overline{R_{N}^{2}}(0) \sim N^{2 / 3}$ and $\overline{R_{N}^{2}}(\epsilon) \sim N$, which implies that $G(x)$ should increase as $x^{1 / 3(1-\Delta)}$ and the cross over value $N^{*}(\epsilon) \sim \epsilon^{-1 / 3(1-\Delta)}$. A direct reliable estimate of $N^{*}(\epsilon)$ is not possible from our data and this scaling theory prediction is difficult to check from our simulations.

\section{B. Average number of visits}

In Fig. 10, we have shown the contour of $\overline{n_{N}}(\mathbf{x})=1$ for an EW of length $N=10^{6}$ steps in the presence of noise with strength $\epsilon=0.01$. The averaging is done over $10^{6}$ initial conditions. The best fit to the data gives the mean radius $\langle R\rangle=296.401 \pm 0.015$, i.e. circular within an error bar of $0.005 \%$. The inset shows the close up of a particular region of the ring. On comparing this with the $\epsilon=0$ case, we see that the mean radius of an EW with $1 \%$ noise strength is about two times greater than the mean radius without noise.

In Fig. 11, we have plotted $\overline{n_{N}}\left(x \mathbf{e}_{x}\right)$ vs $x / N^{1 / 2}$, where $\mathbf{e}_{x}$ is the unit vector along $x$-axis, for an EW in the presence of noise of strength $\epsilon=0.01$ for $N=1 \times 10^{5}, 1 \times 10^{6}$ and $5 \times 10^{6}$ steps. The plot shows that, except near the origin where there is a $\log (N)$ dependence, the data for various $N$ collapse on top of each other.

We also obtain the width, $W_{N}$, of the surface formed by the EW in the presence of noise. We find that $W_{N} \sim N^{1 / 2} \sim R$ as expected for random walks. Hence for nonzero $\epsilon$, the asymptotic shape of the cluster is not circular in individual realizations. Some circular symmetry is only seen in the ensemble averages (i.e., lines of constant $\bar{n}(\mathbf{x})$ ). 


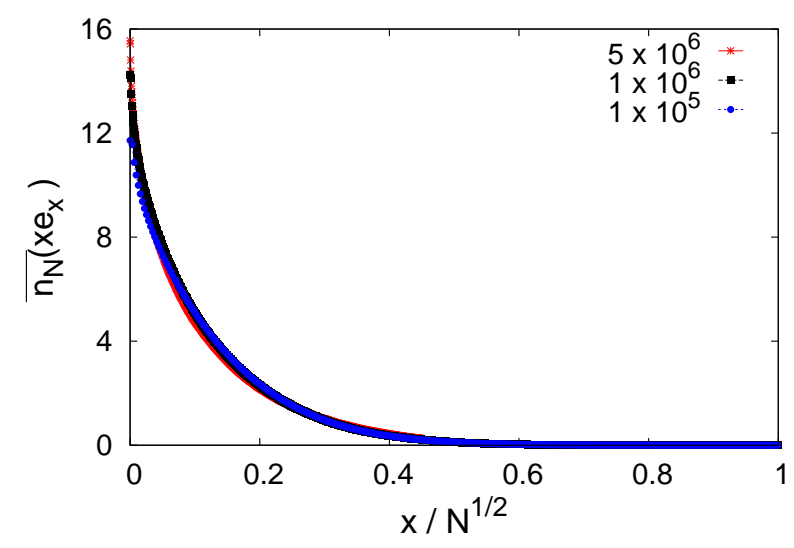

FIG. 11: (Color online) Data collapse of $\overline{n_{N}}\left(x \mathbf{e}_{x}\right)$ vs $x / N^{1 / 2}$ for the EW of $N=1 \times 10^{5}, 1 \times 10^{6}$ and $5 \times 10^{6}$ steps with noise strength $\epsilon=0.01$.

\section{CONCLUSIONS}

We have studied an Eulerian walker on a 2 dimensional square lattice using Monte Carlo simulations. In the absence of noise, the mean square displacement $\left\langle R_{N}^{2}\right\rangle \sim$
$N^{2 / 3}$. We find that lines of constant average number of visits seem to be perfectly circular. This result is not entirely unexpected, given the fact that Eulerian walkers can be considered as a particular type of derandomized random walkers [9]. However, note that the result does depend on the fact that Eulerian walkers in two dimension return to origin infinitely often, and while the $a v$ eraged cluster shape would be expected to show circular symmetry, one does not expect each individual cluster to show spherical shape in dimensions $d>2$.

We also estimated the roughness exponent for the boundary of the visited region. This has a slow convergence to its asymptotic value, but our data is consistent with it belonging to the KPZ universality class. We also find that even a small randomness in the rule for walker's next step changes the asymptotic properties: the mean square displacement shows a crossover from the Eulerian like $\left\langle R_{N}^{2}\right\rangle \sim N^{2 / 3}$ to a simple random walk behaviour $\left\langle R_{N}^{2}\right\rangle \sim N$. In higher dimensions $d>2$, an Eulerian walker does not return to previously visited sites often, and one would expect a random walk like behavior even in the zero-noise case.

DD thanks B. Toth and B. Veto for discussions. We thank J. Propp for a critical reading of the earlier version of the paper.
[1] D. Richardson, Proc. Camb. Phil. Soc., 74, 515 (1973).

[2] T. Vicsek, Fractal Growth Phenomenon (World Scientific, Singapore, 1989) and references therein.

[3] D. Dhar, in On Growth and Form, Ed. H. E. Stanley and N. Ostrowsky, (Martinus Nijhoff, Dordrecht,1986), p 288.

[4] A. F. Boer and F. Redig, J. Stat. Phys. 130, 579 (2008).

[5] D. Dhar, T. Sadhu and S. Chandra, Europhys. Lett. 85, 48002 (2009).

[6] V. B. Priezzhev, D. Dhar, A. Dhar and S. Krishnamurthy, Phys. Rev. Lett. 77, 5079 (1996).

[7] A. M. Povolotsky, V. B. Priezzhev and R. R. Shcherbakov, Phys. Rev. E 58, 5449 (1998).

[8] P. Bak, C. Tang and K. Wiesenfeld, Phys. Rev. Lett. 59, 381 (1987).

[9] A. E. Holroyd and J. Propp, Preprint, arXiv:0904:4507v1 (2009).

[10] S. Angelepoulos, B. Doerr, A. Huber and K. Panagiotouk, Elec. J. Combinatorics 16, R102 (2009).

[11] N. S. Izmailian, V. V. Papoyan, V. B. Priezzhev and C. K. Hu, Phys. Rev. E 75041104 (2007).

[12] L. Levine, The Rotor-Router Model, Harvard University senior thesis, (2002); eprint: math/0409407

[13] M. Kleber, Math. Intelligencer 27, 55 (2005).

[14] L. Levine and Y. Peres, Math. Intelligencer 27, 9 (2005); Indiana Univ. Math J. 57, 431 (2008).

[15] B. Toth and B. Veto, Preprint, arXiv:0803.2848 (2008).

[16] B. Toth, Ann. Probab. 23, 1523 (1995).

[17] E. Grosswald, Representations of Integers as Sums of Squares (Springer-Verlag, Berlin, 1984).

[18] T. Halpin-Healy and Y. C. Zhang, Phys. Rep. 254, 215 (1995).

[19] A. B. Harris, J. Phys. C 7, 1671, (1974).

[20] S. S. Manna, J. Phys. A 24, L363 (1991).

[21] A. Ben-Hur and O. Biham, Phys. Rev. E 53, R1317 (1996).

[22] P. K. Mohanty and D. Dhar, Phys. Rev. Lett. 89, 104303 (2002).

[23] A. Bonachela, J. J. Ramasco, H. Chate, I. Dornic and M. A. Munoz, Phys. Rev. E 74, 050102 (2006).

[24] S. G. Lee and S. B. Lee, Phys. Rev. E 80, 011106 (2009). 